\title{
Teaching Arabic Sign Language through an Interactive Web based Serious Game
}

\author{
Elaachak Lotfi \\ Computer science, systems \\ and telecommunication \\ Laboratory (LiST) \\ Faculty of Sciences and \\ Technologies \\ Abdelmalek Essaadi University \\ Tangier, Morocco
}

\author{
Belahbib Amine \\ Computer science, systems \\ and telecommunication \\ Laboratory (LiST) \\ Faculty of Sciences and \\ Technologies \\ Abdelmalek Essaadi University \\ Tangier, Morocco
}

\author{
Bouhorma Mohammed \\ Computer science, systems \\ and telecommunication \\ Laboratory (LiST) \\ Faculty of Sciences and \\ Technologies \\ Abdelmalek Essaadi University \\ Tangier, Morocco
}

\begin{abstract}
The tools of teaching have known a big evolution with the integration of the new technology in the learning process, especially the serious games, thus the appearance of several input devices, e.g. "leap motion controller, kinect, etc.", has expanded the scope of the users which reaches people with disabilities e.g. "blind, deaf, etc.", and that use this kind of video games. This paper will detail the development of a web based serious game dedicated for deaf children, in order, that they learn the Arabic sign alphabet, through both an input device called leap motion controller and hand gesture recognition based in the artificial neural network. The proposed serious game will be integrated in a learning management system via the use of the Scorm standards, in order to make the course on e-learning platform more interactive.
\end{abstract}

\section{General Terms}

Arabic Sign Languages, Serious games, E-learning

\section{Keywords}

Serious Games, Scorm, e-learning, ANN, Sign Language

\section{INTRODUCTION}

More than two hundred million inhabitants of 22 countries across the Middle East and North Africa speak Arabic as native language [1]. A significant proportion of them are deaf, causing several communication problems with each other and also with people who speak the language. For this reason there is a set of sign languages called Arabic sign languages (ARSLs), but unfortunately ARSLs are still in their developmental stages. Only in these last years has there been an awareness of the existence of communities consisting of individuals with disabilities; the Deaf people are not an exception. Arab Deaf communities are almost closed ones. Interaction between a Deaf community and a hearing one is minimal and is basically concentrated around families with deaf members, relatives of the deaf, and sometimes play friends and professionals [2], for this a need appeared to unify Arabic sign language in all Arab countries. This derived the Council of Arab Ministers of Social Affairs (CAMSA) to take a decision of developing a unified Arab sign language dictionary and publish it to all countries, in an attempt to help Arab deaf people to have a common language in addition to their local language [3].

To cope with these problems, it's important to encourage the little children with this handicap to learn the Arabic sign language, which will standardize communication despite the existence of different dialects in the Arab world. For this reason the best way to teach this language is the learning via serious games, thanks to their benefits, like interactivity, transfer of knowledge in a fun and attractive way. In addition the integration of input devices like "kinect, leap motion controller, etc." will allow this segment of special users to pass a good and beneficial time by playing the video games based on the gestures of their hands.

This paper presents the development of a web-based serious game composed of two levels; the first one is in $2 \mathrm{~d}$ environment by cons the second one is in $3 \mathrm{~d}$ environment. The main objective of the proposed serious game is to teach the deaf learners the basics of the Arabic sign language through an input device called leap motion controller that detects both hand and fingers coordinates. These coordinates will feed the artificial neural network that will recognize the hand gesture performed by the learners. The proposed serious game will be integrated into a learning management system via the Scorm standards, in order to enrich the course and to create a variety of choices for the learners.

\section{RELATED WORK}

The apparition of several interactive input devices, e.g. "kinect, leap motion controller, etc." has expanded the scope of serious games, in order to cover other segments of users, e.g. "deaf and blind people", with the advantages of the serious games and the interactive input device, this special segment of learner will be able to learn in a fun way and live a beneficial experience. Until today several sign language video games have been developed by different laboratories and institutions. A team from the Superior Institute of Porto has developed a serious game which the learner controls a character and interacts with various objects and non-player characters with the aim of collecting sever gestures from the Portuguese Sign Language [4].Another realization of the team from Nova University of lisboa, that has developed a serious game "Kinect-Sign " composed of two modes: School-mode and Competition mode. The first provides a school-like environment where the learner learns the letter-signs and the second provides the user an environment used towards testing the learned skills. This serious game is based on the creation of a gesture library, relying on the Kinect depth camera; and 2 the real-time recognition of gestures, by comparing what the depth camera information to the existing gestures previously stored in the library [5]. The number of the serious games dedicated for deaf and dumb learners, is insufficient for the large number of the people who suffer from this handicap and especially for the learners from Arabic countries. 
In this perspective of research and development this paper will present the development of a web-based serious game that teaches deaf kids the Arabic sign language through the leap motion controller.

\section{THEORETICAL BACKGROUND}

\subsection{Arabic Sign Language}

Arabic sign languages (ArSL) are not particularly different from other known sign languages, such as ASL and BSL. In fact, the Arabic varieties in use have undergone some lexical influence from other sign languages [6]. ArSL is limited to represent nouns, adjectives and verbs. Prepositions and adverbs are represented in the context of articulation by specifying locations, orientations and movement. Intensifiers represented by iteration [7]. There are 29 hand gestures related to each Arabic sign alphabet as shown in Fig 1.

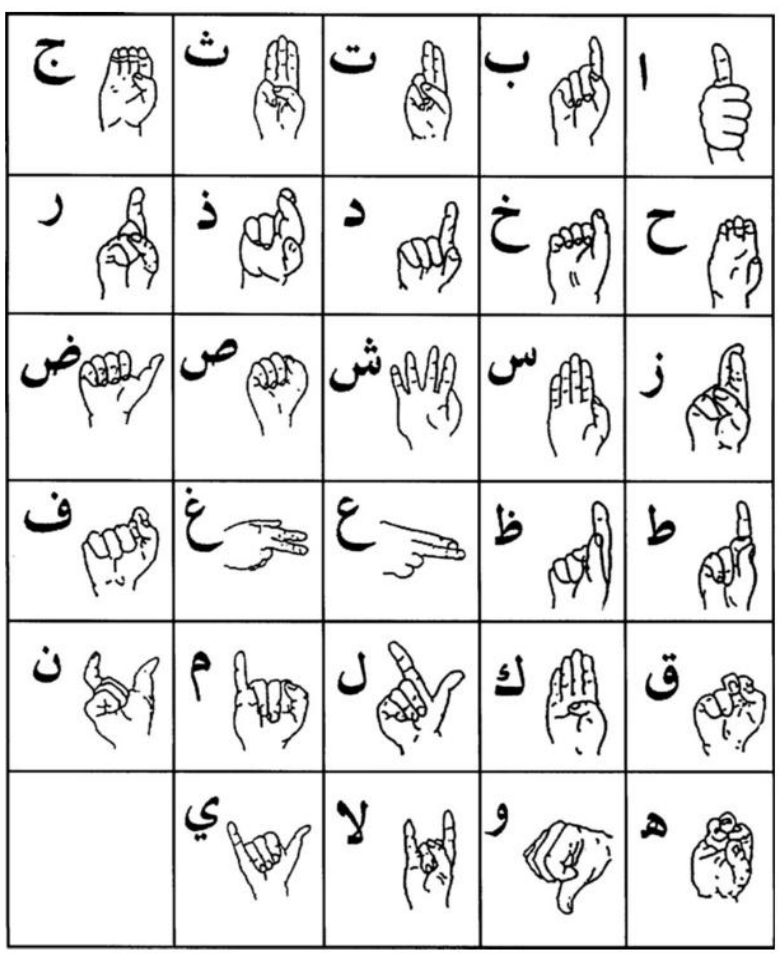

Fig 1: Arabic Sign Alphabets

\subsection{Artificial Neural Network}

Artificial Neural Network (ANN) is a field of Artificial Intelligence that allows finding the data structures and algorithms for learning and classification of data, by inspiration from the human brain. The ANNs have been developed as generalizations of mathematical models of biological nervous systems. A first wave of interest in neural networks (also known as connectionist models or parallel distributed processing) emerged after the introduction of simplified neurons [8].

The basic elements of the neural networks are called artificial neurons, or simply neurons or nodes. All signals inside the network can be 1 or -1 . The neuron calculates a weighted sum of inputs and compares it to a threshold. If the sum is higher than the threshold, the output is set to 1 , otherwise to -1 . A typical artificial neuron and the modeling of a multilayered neural network are illustrated in Fig 2.

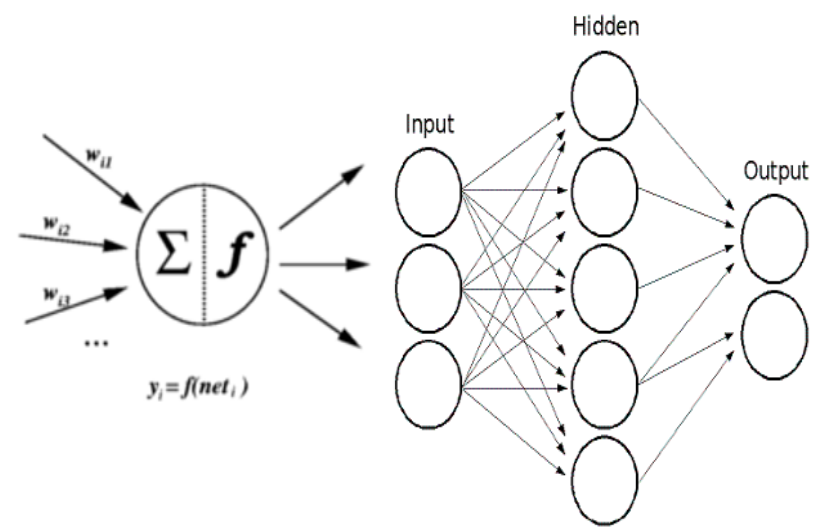

Fig 2: Architecture of an artificial neuron and a multilayered neural network.

The signal flow from the inputs is considered to be unidirectional "in one direction". The neuron output signal O is given by the following relationship:

$$
O=f(n e t)=f\left(\sum_{i=1}^{n} w \mathrm{i} * \mathrm{xi}\right)
$$

Where wi is the weight vector, and the function $f$ (net) is referred to as an activation function. The variable net is defined as a scalar product of the weight and input vectors.

$$
\text { net }=w T x=w 1 \times 1+w 2 \times 2+\cdots+w n \times n
$$

Where $\mathrm{T}$ is the transpose of a matrix, The output value $\mathrm{O}$ is computed as:

$$
O=f(\text { net })=\left\{\begin{array}{lr}
1, & \text { if } w T x \geq \theta \\
0, & \text { otherwise }
\end{array}\right.
$$

Where $\theta$ is called the threshold level; and this type of node is called a linear threshold unit.

The most common architecture used to implement a neural network is composed of three layers the input, hidden, and output layers. . In neural networks, the signal direction begins from the input towards the output elements, through one or several hidden layers of neurons followed by an output layer of linear neurons. Multiple layers of neurons with nonlinear transfer functions allow the network to learn nonlinear relationships between input and output vectors. The Perceptron is a single layer neural network whose weights and biases could be trained to produce a correct target vector when presented with the corresponding input vector [9]. One of the most important features of artificial neural networks is their ability to learn. There are many different algorithms that can be used when training ANNs, each with their own advantages and disadvantages. The learning process within artificial neural networks is a result of altering the network's weights, with some kind of learning algorithm, e.g."Perceptron Learning algorithms, Back-Propagation, Bayesian Learning”.

In this paper, the algorithm chosen for learning the Perceptron is the Gradient Descent algorithm. The Gradient Descent is an algorithm that involves finding the minimum of a complicated nonlinear function called "error function". This function describes the error that the neural network makes in approximating or classifying the training data, as a function of the weights of the network. The main objective is to reduce error in order to become as small as possible and should thus 
try to move towards the point of minimum error [10]. The delta rule [Gradient Descent] algorithm described below:

- $\quad$ Initialize $\mathrm{w}=$ small random numbers

- Until termination condition is met (error bound, or iterations of training examples)

$\circ \quad$ Initialize all $\Delta$ wi $\leftarrow 0$

- For each training example $(x, t)$

- Compute the output of each node: $\mathrm{O}(\mathrm{x})$

- For each weight unit wi: $\Delta$ wi $\leftarrow \Delta \mathrm{wi}+\eta(\mathrm{t}-\mathrm{o}) \mathrm{xj}$

$\circ \quad$ For Each weight unit wi: wi $\leftarrow$ wi $+\Delta$ wi

Among the feature of data rule [Gradient Descent] algorithm:

- Attempts to minimize the squared error of the training examples

- Guaranteed that there is a single minimum error

- Uses Sigmoid function $\sigma(\mathrm{x}): 1 /(1+\mathrm{e}-\mathrm{x})$

- $\quad \mathrm{d} \sigma(\mathrm{x}) / \mathrm{dx}=\sigma(\mathrm{x})(1-\sigma(\mathrm{x}))$

- The sigmoid function is used because the derivative is very easy to find

- Gradient: $\nabla \mathrm{E}[\mathrm{w}]=[\mathrm{dE} / \mathrm{dwo}, \mathrm{dE} / \mathrm{dw} 1, \mathrm{dE} / \mathrm{dw} 2, .$. , $\mathrm{dE} / \mathrm{dwn}]$

- Training Rule: $\Delta \mathrm{w}=-\eta \nabla E[\mathrm{w}]$

\subsection{Technical standard for e-learning software SCORM}

The Sharable Content Object Reference Model (SCORM) was created to help the reusability, interoperability, portability, access, maintenance and adaptation of Learning Objects [11]. It's a collection of standards and specifications that allows the research, the importation and the integration of the content in several learning platforms e.g. "LMS, CMS, LCMS “.The Learning Objects must be created to the SCROM Standards to ensure interoperability across the different tools and elearning platforms [12] see the Fig.3.

The SCORM standards are XML-based industry formalization and the high level requirements defined for learning contents in SCORM are content reusability, accessibility, durability, and interoperability. SCORM enables the reuse of web-based learning content across multiple environments and products. Clearly, it is not a model for learning content; but it is a model for content delivery [13].

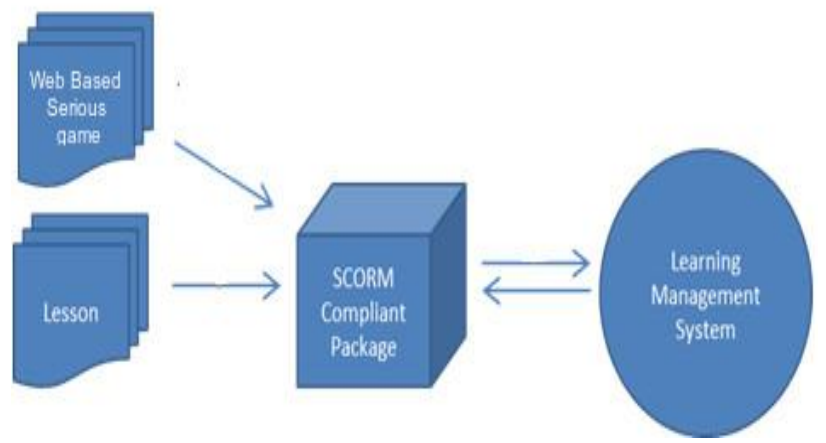

Fig. 3: Integration of SCORM Standard with LMS

\section{HAND GESTURE RECOGNITION}

As motioned before the proposed serious game will be equipped by an input device called leap motion controller that will track the hand gestures "palm and finger positions in 3d coordinate", and with the neural network that will recognize the hand gesture of the player. The gesture recognition process diagram is illustrated in fig 4 .

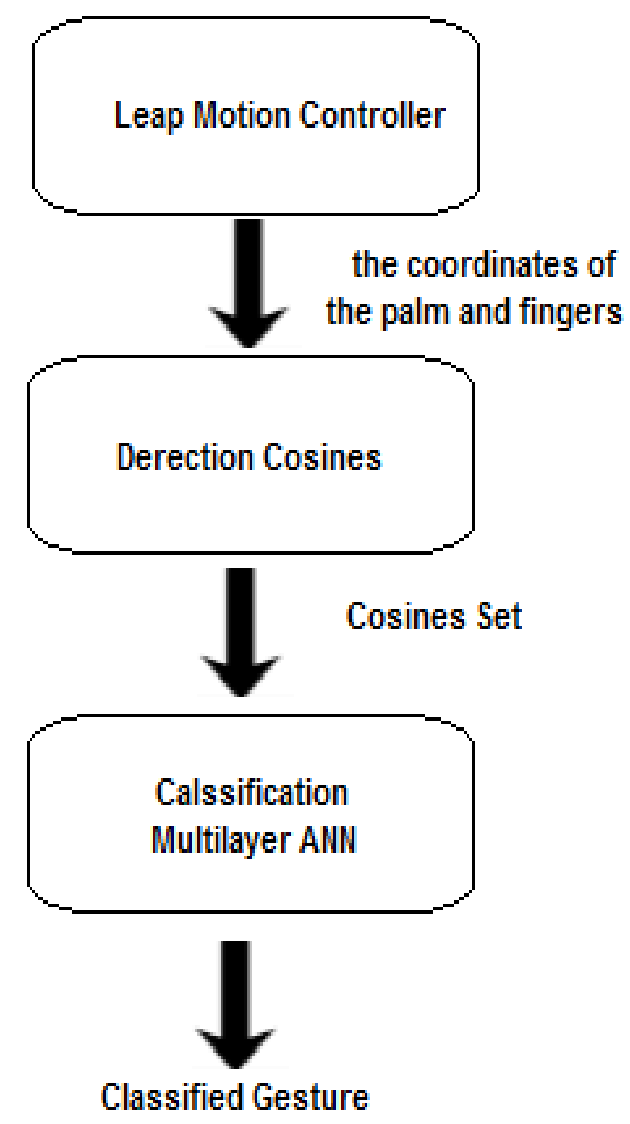

Fig. 4: Block diagram of the recognition system

\subsection{Leap motion controller}

The Leap Motion controller is a small device that can be connected to a computer using a USB. It uses infrared (IR) imaging to determine the position of predefined objects in a limited space in real time. It can then sense hand and finger movements in the air above it, and these movements are recognized and translated into actions for the computer to perform. According to the official information founded in the official web site of leap motion [14], the Leap software analyzes the objects observed in the device's field of view. It recognizes hands, fingers, and tools, reporting discrete positions, gestures, and motion. The controller's field of view is an inverted pyramid centered on the device Fig. 5. The effective range of the controller extends from approximately 25 to 600 millimeters above the device. The controller itself is accessed and programmed through Application Programming Interfaces (APIs), with support for a variety of programming languages, ranging from $\mathrm{C}++$ to Python and JavaScript. The positions of the recognized objects are acquired through these APIs. The Cartesian and spherical coordinate systems used to describe positions in the controller's sensory space. 


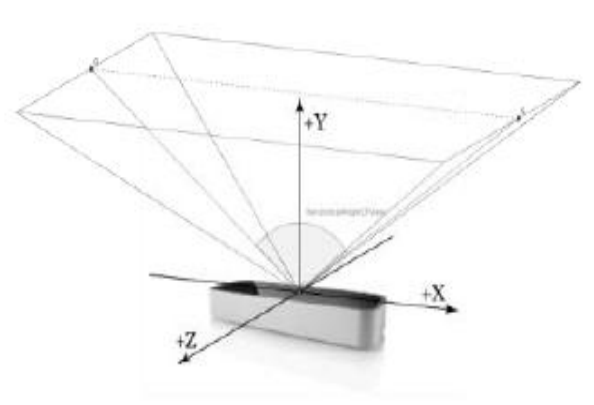

Fig. 5: Leap Motion's Field Of View

With the features that leap motion controller offers and with the use JavaScript application programming interface, booth integrated it in the proposed video game, therefore, the player has to move his hand and catch the random generated objects in order to drag and drop them, then place them in a correct container. With this possibility the proposed video game will become more interactive and so close to the real case. That will create envy into the player to play more. In addition, this concept will allow us to save all gestures done by the players during a sequence of video game; this data will be used by educational data maiming to understand the player's behaviors and also to analyze their performances.

\subsection{The hand gesture tracking}

Among the features of the leap motion controller is the tracking of the hand gestures by giving the $3 \mathrm{~d}$ coordinate " $x$, $y, z$ " of the palm and tip of each finger as in Fig.6.

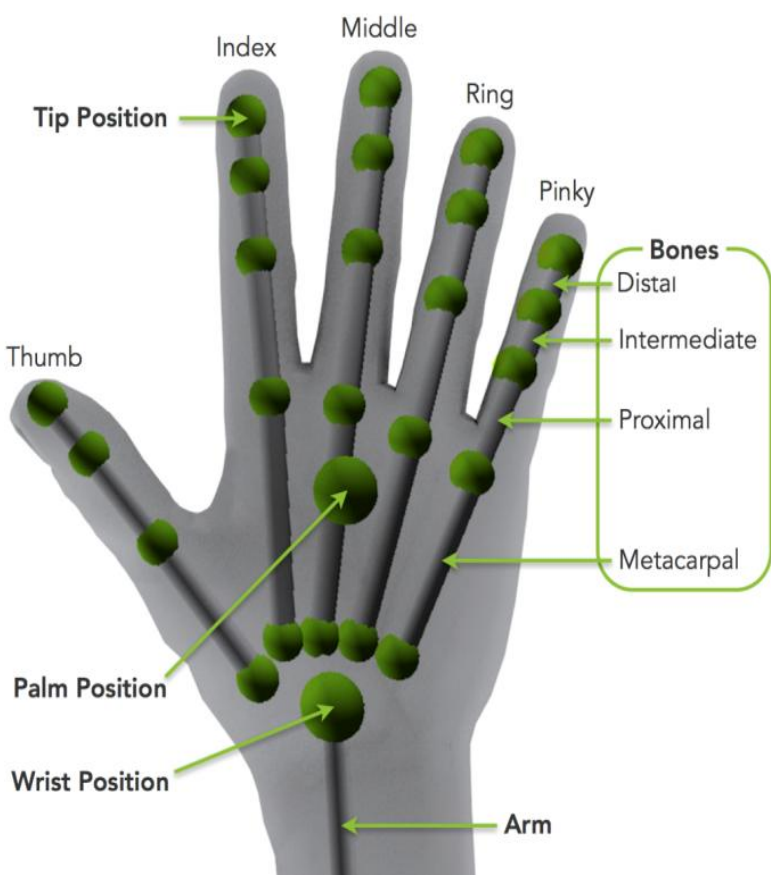

Fig. 6: Palm and Tip Positions given by the leap motion controller

Thereafter, these coordinates will be used to calculate the direction cosines of each vector that will be constructed according to the $3 \mathrm{~d}$ coordinates of the palm position and the $3 \mathrm{~d}$ coordinates of the tip position of each finger:

$$
\underline{\mathrm{A}}=A_{x} \underline{\mathrm{i}}+A_{y} \underline{\mathrm{j}}+A_{z} \underline{\mathrm{k}}
$$

Magnitude of a Vector:

$$
A=\sqrt{A_{x}^{2}+A_{y}^{2}+A_{z}^{2}}
$$

Direction Cosines:

$$
\begin{aligned}
& \cos (\alpha)=\frac{A_{x}}{A}, \quad \cos (\beta)=\frac{A_{y}}{A}, \quad \cos (\gamma)=\frac{A_{z}}{A}, \\
& \cos ^{2}(\alpha)+\cos ^{2}(\beta)+\cos ^{2}(\gamma)=1
\end{aligned}
$$

The direction cosines of each vector, in our case the five vectors related to the tip of each finger and the palm that will feed the input of the neural network Fig.7 that will be intended to recognize the hand gesture of the learner. The input layer is composed of 15 nodes, each node is fed by Cosine values, and the set of the three cosines represent the direction cosines of each finger. The hidden layer is composed of four nodes, and in the output layer there is one node that will give either 1 or 0 . The Table 1 describes some characteristics of the used neural network.

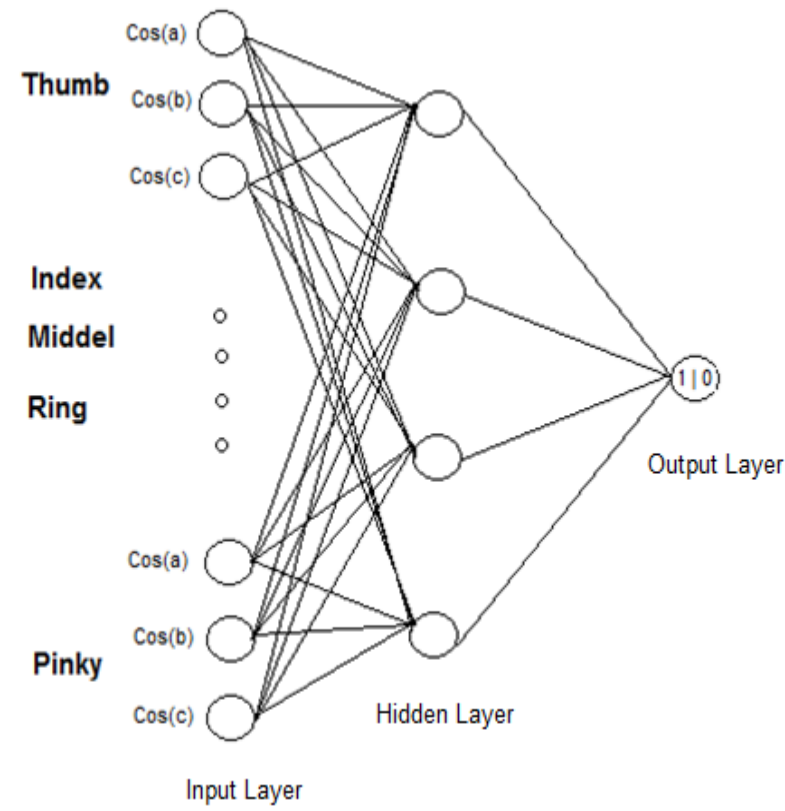

Fig. 7: The neural network of hand gesture recognition

Table 1. Characteristics of the artificial neural network

\begin{tabular}{|c|c|}
\hline Element & value \\
\hline Input layer & 15 nodes \\
\hline Hidden layer & 4 nodes \\
\hline Output layer & 1 node \\
\hline Activation function & Sigmoid \\
\hline Learning algorithm & Gradient Descent \\
\hline
\end{tabular}




\begin{tabular}{|c|c|}
\hline Threshold & 0.9 \\
\hline Training error Threshold & 0.0001 \\
\hline Training iteration & 20000 \\
\hline Learning rate & 0.1 \\
\hline
\end{tabular}

\section{ARABIC SIGN LANGUAGE SERIOUS GAME}

The proposed web-based serious game was developed by using several JavaScript APIs, e.g. "2d, 3d video game engines, ANN JavaScript API, etc.", in order that the video game will be run in different web browsers. The game architecture, detailed in Fig 8 is composed of different modules related to each others, with the aim to make a full system that begins by tracking the hand gesture "coordinates, direction cosines" until the hand gesture recognition and the evaluation of the sign performed by the learner. This web based video game is composed of two game levels; each level has specific characteristics such as in the level one the environment of the game is $2 \mathrm{D}$ against level two is $3 \mathrm{D}$. Level one will be played by using an input device "leap motion controller" based on hand gesture that will allow deaf learners to perform several hand gestures that will match the Arabic letters displayed on the screen.

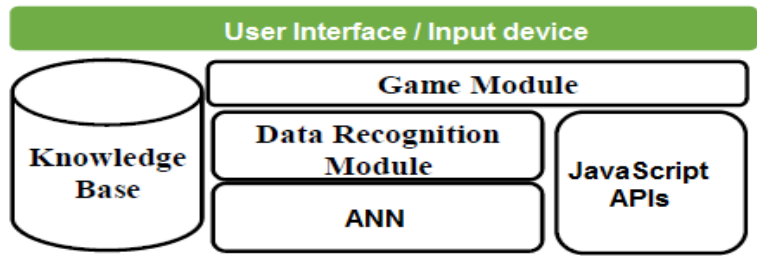

Fig.8: web based video game architecture

The scenario of the level one Fig.9 is simple the learner has to perform the sign that will match the letter displayed on the screen, for each good answer the learner will win some points that will be added in his score, otherwise, he will lose points from his score. The learner must respect the time "three minutes" and try to increase his score by giving the right response.

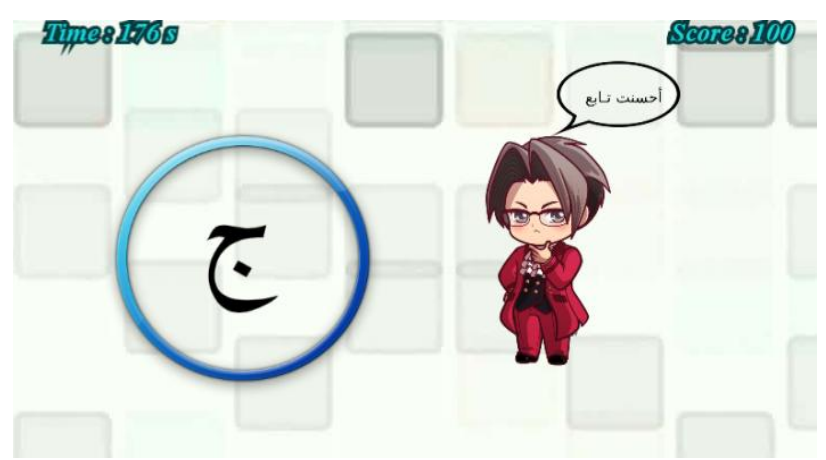

Fig.9: screenshot from the level one of the proposed 2D web based serious game

The level tow Fig.10 of the proposed serious game is in 3D, it's developed according to the First-person shooter genre. The main objective of this level is letting the learner to live another experience different from the level one, that will create a desire into him to continue playing the video game, in this level the learner must find all the cubs that randomly move in the maze, the number of the cubs depend on the picture displayed on the screen, because each cube represents a sign, with the collection of these cubs in the right order the player will construct the name of the object "picture" displayed on the screen, to make the game more challenging the developers have added in this level some obstacles that the learner must avoid. In general, the learner loses the game when the score becomes 0 . When the learner collects the cubes in the right order other pictures will be randomly displayed sulfide as his progress in the game.

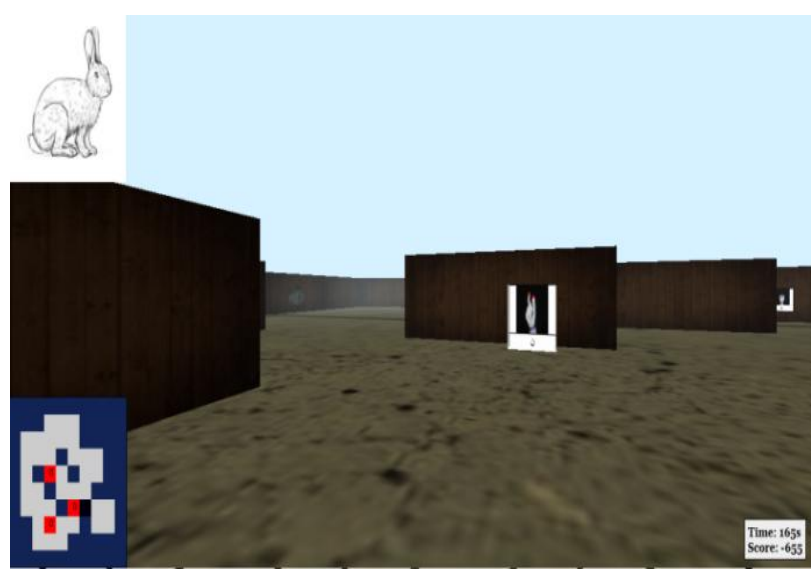

Fig.10: screenshot from the level two of the proposed 3D web based serious game

\section{RESULTS}

The development process has led to the realization of a web-based serious game composed of two levels, the first one is in a two-dimensional environment, equipped with an input device that tracks the hand gesture to extract the coordinates between the palm position and each tip position, these coordinates will be used to calculate the direction cosines, each calculated cosine will feed the inputs of the neural network, in order to recognize the hand gesture performed by the learner, then the recognized gesture will be checked if it matches the letter displayed on the screen. The second level is in a third dimensional environment, this level adopts the game genre called the First person shooter (FPS), the diversity of environments will allow the learner to support a diverse experience that will create a desire in him to play.

As mentioned before the neural network who will take care of hand gesture recognition, is integrated into the proposed web based serious game "level one", this neural network is a multilayer Perceptron with activation function "sigmoid", the input layer of the proposed neural network is composed of fifteen nodes, four nodes as hidden layer, and one node as output layer. The learning algorithm used to train the network is the Gradient Descent, with 20000 training iteration, 0.1 as the value of the learning rate and threshold is 0.9 .

Some of the results given by the neural network are detailed in the Table 2 e.g. "the first four Arabic sign language". To explain more the algorithm used to match a performed gesture made by the learner with the letter that corresponds to it, the neural network gives the approximate values calculated since the gesture performed by the learner and the 29 patterns "correspond to each Arabic letter", afterwards the algorithm selects the max value from the set of the calculated values, the order of the chosen value corresponds to the order of the chosen letter, in this way the algorithm implemented in the web-based serious game recognizes the gesture performed by 
the learner than evaluate it to add some points to the global score or vice versa.

Table 2. The values given by neural network concerning the performed gesture and some patterns "four first Arabic letters"

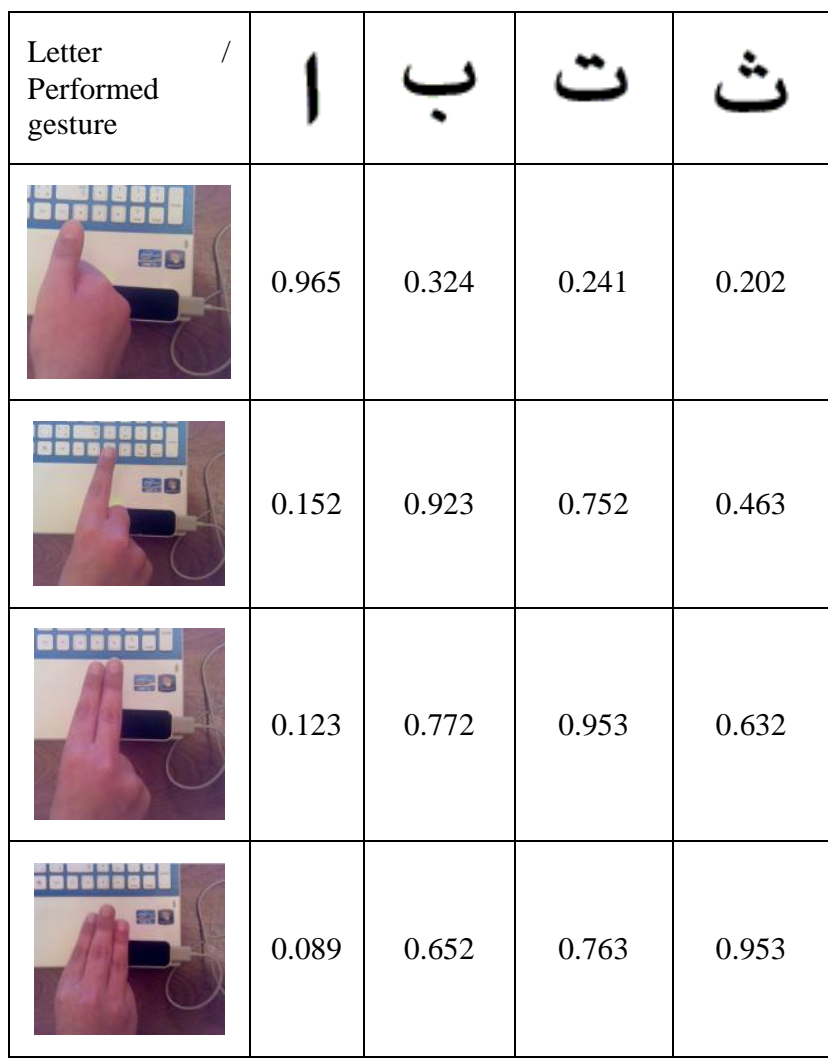

The proposed web-based serious game was integrated in a learning management system (LMS) "moodle" [15] "Fig11, 12", via the Scorm standards designed to enable the interoperability, accessibility, and reusability of web-based learning content. The main objective of the integration of the web based serious game into LMS is to add more interactivity in the course and also change the way of learning by introducing an entertaining tool that will attract attention of the learner.

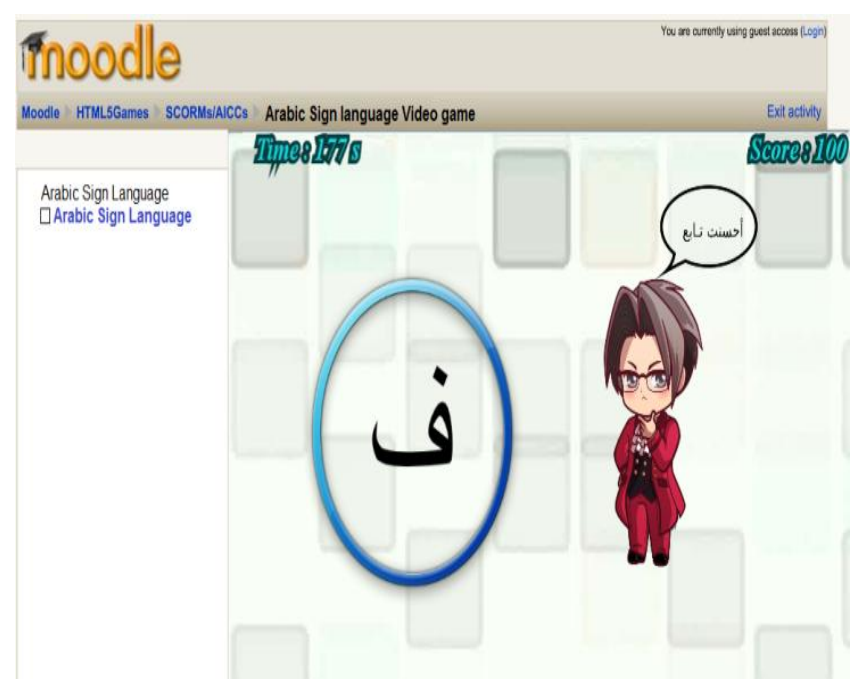

Fig.11: screenshot from the level one integrated with Scorm in to the Moodel LMS

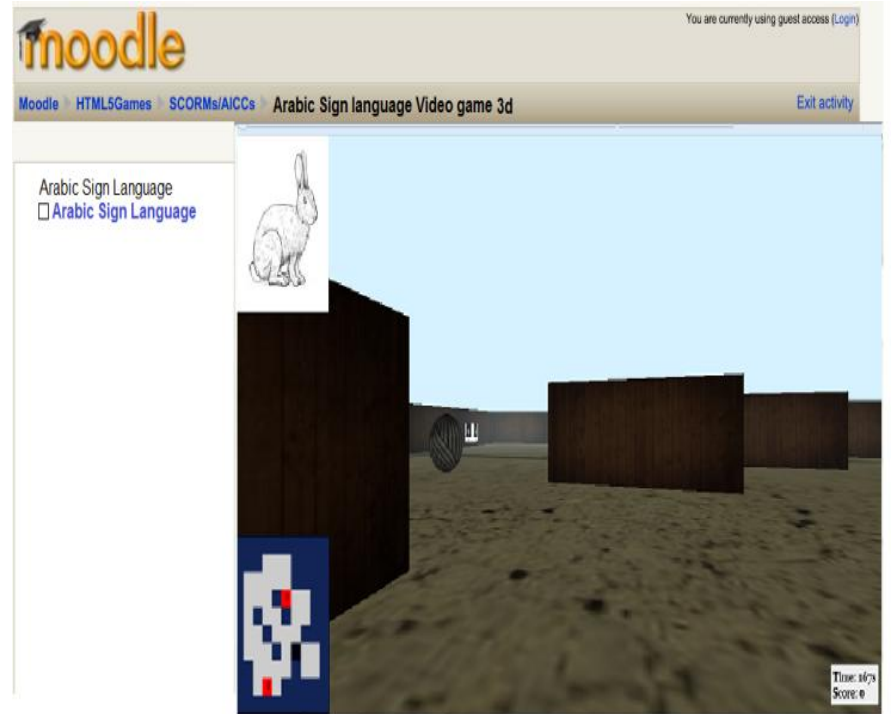

Fig.12: screenshot from the level two integrated with scorm in to the Moodel LMS

In order to value the full system, the proposed game was given to 10 deaf kids to evaluate their level of learning by saving the time and the score of each learner, according to the three game attempts Table 3.

Table 3. The learner outcomes since the three attempts of the level one of the web-based serious game

\begin{tabular}{|l|c|c|c|c|c|c|}
\hline & \multicolumn{2}{|c|}{1 Attempt } & \multicolumn{2}{c|}{2 Attempt } & \multicolumn{2}{c|}{3 Attempt } \\
\hline & Time & Score & Time & Score & Time & Score \\
\hline Learner 1 & $180 \mathrm{~s}$ & 150 & $180 \mathrm{~s}$ & 210 & $180 \mathrm{~s}$ & 250 \\
\hline Learner 2 & $60 \mathrm{~s}$ & 0 & $180 \mathrm{~s}$ & 100 & $180 \mathrm{~s}$ & 190 \\
\hline Learner 3 & $180 \mathrm{~s}$ & 130 & $73 \mathrm{~s}$ & 0 & $180 \mathrm{~s}$ & 125 \\
\hline Learner 4 & $130 \mathrm{~s}$ & 0 & $70 \mathrm{~s}$ & 0 & $180 \mathrm{~s}$ & 100 \\
\hline Learner 5 & $180 \mathrm{~s}$ & 200 & $180 \mathrm{~s}$ & 250 & $180 \mathrm{~s}$ & 220 \\
\hline Learner 6 & $50 \mathrm{~s}$ & 0 & $140 \mathrm{~s}$ & 0 & $180 \mathrm{~s}$ & 10 \\
\hline Learner 7 & $180 \mathrm{~s}$ & 30 & $170 \mathrm{~s}$ & 0 & $180 \mathrm{~s}$ & 90 \\
\hline Learner 8 & $180 \mathrm{~s}$ & 25 & $180 \mathrm{~s}$ & 105 & $180 \mathrm{~s}$ & 65 \\
\hline Learner 9 & $30 \mathrm{~s}$ & 0 & $180 \mathrm{~s}$ & 55 & $175 \mathrm{~s}$ & 0 \\
\hline Learner 10 & $180 \mathrm{~s}$ & 45 & $180 \mathrm{~s}$ & 100 & $180 \mathrm{~s}$ & 120 \\
\hline
\end{tabular}

According to the results given in the Table 3, most learners have known an evolution of their level of learning, the score increase from an attempt of the game to another. This evolution of the learning level depends from a learner to another, the improved level of learning in the learner depends on several factors include his ability of analysis, his way of performing the gesture, his speed, his concentration, his ability to interact with the game, his motivation and in 
addition of other factors that can influence on his performance.

\section{CONCLUSION}

This paper has discussed the development of a web-based serious game dedicated for deaf kids to learn Arabic sign language, the use of the input device has changed the traditional method to play a video game that often exploits the keyboard or the mouse, with this new device the game has become more interactive and more beneficial, because the learner can perform several hand gestures and he can correct them if he performed them in a wrong way, in addition, this method of learning based on serious game has allowed the learners to increase their level of learning and allowed them to experience a new experience different from the traditional course that they are accustomed to follow. Concerning the future research works, the research team will integrate other levels of the game, in order, that the learners learn the Arabic numbers and some special signs concerning the communication. And also integrate other devices e.g. "kinect" in the proposed serious game to allow learners to experiment these devices, and their interaction with serious games.

\section{ACKNOWLEDGMENTS}

Our thanks to the students and all the other participants who have contributed towards contribution of the paper.

\section{REFERENCES}

[1] Abdel-Fattah, M. (2005). Arabic sign language: A perspective. Journal of Deaf Studies and Deaf Education, $10,212-221$

[2] M. A. Abdel-Fattah, Arabic Sign Language: A Perspective, Journal of Deaf Studies and Deaf Education vol. 10 no. 2, Department of Languages and Translation, Birzeit University, 2005.

[3] Paula E, Nuno E, Rosa R, F.P. M, José Bidarra, Ana Bela B, Pedro R, Jorge L, Marcelo N, Vaz De C. Serious Game for Sign Language. International conference on Intelligent Systems, Data Mining and Information Technology (ICIDIT'2014), April 21-22, 2014 Bangkok Thailand.

[4] P.V. Paul, Language and Deafness, Jones \& Bartlett Learning, 2008.
[5] João Gameiro, Tiago Cardoso, Yves Rybarczyk. Kinect-Sign: Teaching Sign Language to "Listeners" through a Game. eNTERFACE 2013: 141-159.

[6] Miller, C. (1996). Disc: Arabic Sign Language, Re: 7.1101. Retrieved March 14, 2004, online access: http://www.linguislist.org/issues/7/7-1110.html.

[7] M.A. Abdel-Fattah, "Arabic Sign Language: A Perspective," Journal of Deaf Studies and Deaf Education, vol. 10, Spring. 2005, pp. 212 -221.

[8] McCulloch, W.S. and Pitts, W.H. (1943) A Logical Calculus of the Ideas Immanent in Nervous Activity. Bulletin of Mathematical Biophysics,5, 115-133.

[9] A.Ajith, Artificial Neural Networks, Handbook of Measuring System Design, edited by Peter H. Sydenham and Richard Thorn. (2005) John Wiley \& Sons, Ltd. ISBN: 0-470-02143-8.

[10] Er. Parveen Sehgal, Dr. Sangeeta Gupta, Dr. Dharminder Kumar, "AStudy of Prediction Modeling Using Multilayer Perceptron (MLP) With Error Back Propagation", Proceedings of AICTE sponsored National Conference on Knowledge Discovery \& Network Security: February 2011, ISBN: 978-81-8465-755-5, pp. 17-20.

[11] Gonzalez-Barbone, V., Anido-Rifon, L.: From SCORM to Common Cartridge: A step forward. Computers \& Education Volume 54, Issue 1, January 2010, Pages 88102 (2009).

[12] ADL Technical Team.: ADL Guidelines for Creating Reusable Content with SCORM 2004 (2008) Online at: http://www.adlnet.org. site visited (6/2/2014).

[13] H. SRIMATHI ， S.K. SRIVATSA. DESIGN OF VIRTUAL LEARNING ENVIRONMENTUSING SCORM STANDARDS. Journal of Theoretical and Applied Information Technology. Vole 4, $\mathrm{N}^{\circ}$ 6, 2008.

[14] Leap Motion Controller. Available online: https://www.leapmotion.com (accessed on 21 October 2014).

[15] Moodle - Open-source learning platform Online: https://moodle.org (accessed on 21 October 2014). 